\title{
Einkaufsentscheidung im Krankenhaus Ohne Rückkopplung mit Ärzten geht es nicht
}

\begin{abstract}
Die Sana Kliniken AG ist die drittgrößte private Klinikgruppe Deutschlands. In 47 Krankenhäusern werden 1,82 Millionen Patienten von 27350 Mitarbeitern versorgt, darunter rund 3800 Ärzte und Ärztinnen, die ein breites Spektrum an medizinischen Fachgebieten und Spezialdisziplinen abdecken. Entsprechend hoch ist der Bedarf an Arzneimitteln, Medizinprodukten, Technik und anderen Leistungen und Produkten. Über den Einkauf und die ausgelagerte Beschaffung und Logistik in der Dienstleistungsgesellschaft Clinic.Log werden jährlich Waren im Wert von über $\mathbf{4 0 0}$ Millionen Euro für die verschiedenen Standorte der Klinikgruppe organisiert. Das Beschaffungsmanagement verantwortet Adelheid Schäfer-Jakobs. Sie ist seit Dezember 2014 Generalbevollmächtigte für Einkauf und Logistik.
\end{abstract}

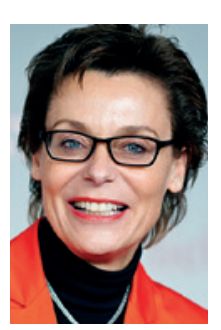

Adelheid Schäfer-Jakobs
Früher wurden Entscheidungen über die Anschaffung medizinischer Bedarfsgüter oder neuer Geräte meist dezentral von Ärzten getroffen, heute wird der Einkauf immer stärker zentralisiert. Trifft das auch für die Sana Kliniken zu, Frau Schäfer-Jakobs? Schäfer-Jakobs: Ja, das trifft auch auf Sana zu, aber nicht erst seit ich in den Klinikverbund gekommen bin. Die Sana Kliniken etablierten die Standardisierung und Bündelung großer Volumina zunächst in den Herzzentren. Später folgten orthopädische Implantate und weitere Standardartikelkataloge. Inzwischen geht es aber längst nicht mehr um die Frage von „zentral versus dezentral“. Das ist wie eine Schwarz-Weiß-Betrachtung, die dem komplexen Geschehen von Qualität, Patientenanforderungen, Markt und Kosten nicht gerecht wird. Es geht vielmehr darum, medizinisch fundierte Entscheidungen und das Wirtschaftlichkeitsgebot in einem Prozess zu verbinden, sodass im Ergebnis die bestmögliche Lösung entsteht.

\section{? Welche Aufgaben kommen dabei auf Sie zu?}

Schäfer-Jakobs: Für die Akzeptanz und die Umsetzung im Krankenhaus braucht es eine führende Verantwortlichkeit, welche die genannten Ziele bei einer möglichst hohen Zufriedenheit der beteiligten Ärzte erreicht. Eine solche Aufgabe ist sehr anspruchsvoll und benötigt kluge Ideen für die Moderation. Ich sehe in der zentralen Führung mit dezentraler Einbindung - vor allem der Ärzte - die Schlüssel zur Qualitätssicherung, Wirt- schaftlichkeit und Mitarbeiterzufriedenheit.

? Wann und wie binden Sie Ärzte in den Entscheidungsprozess ein? Schäfer-Jakobs: Wir binden Ärzte bei allen leistungssensiblen Produkten in die Entscheidungsprozesse ein. Wir wollen erfahren, wie sie die Trends am Markt einschätzen und die Qualität und Kosten der Produkte gewichten, welches Evidenzlevel dem zugrunde liegt und welche Präferenzen bestehen. Die Aufzählung zeigt, dass es sich um einen laufenden Kommunikationsprozess handeln muss, für den wir Fach- und Arbeitsgruppen eingerichtet haben. Wir brauchen Vertrauen, das erst durch professionelle Zusammenarbeit, Verlässlichkeit und Kompromissfähigkeit in Einzelsituationen entstehen kann.

Können Sie dies an einem Beispiel deutlich machen?

Schäfer-Jakobs: Massenmedizinprodukte, wie Handschuhe oder Verbandsmaterial, werden zunächst in Praxistests im Klinikalltag erprobt und bewertet. Diese Bewertungen fließen dann in unsere Kaufentscheidungen ein. Für den Implantate-Einkauf werden in regelmäßigen Treffen mit den Fachexperten die Beschaffungsstrategien für die jeweiligen Produktbereiche festgelegt. Bei medizinischen Großgeräten, werden nach den Vorgaben der Anwender geeignete Geräte evaluiert und in einer gemeinsamen Feinabstimmung ausgewählt.

Welche Entscheidungsautonomie bleibt in den Kliniken und Fachabteilungen?

Schäfer-Jakobs: Nach wie vor entscheiden Ärzte, welches Produkt oder welche
Technik aus einem Bündel verschiedener Alternativen für den individuellen Patienten zum Einsatz gelangt. Auch die medizinische Qualitätssicherung kann nur im Zusammenspiel mit der Anwendung selbst stattfinden, sonst ist keine Evaluation möglich, die wiederum Grundlage für neue Entscheidungen ist. Letztendlich können die beschriebenen Prozeduren nur dann zum Erfolg führen, wenn die Fachlichkeit der Kliniken in die Entscheidung einfließt und die Themen, die im Einkauf und der Beschaffungsorganisation anstehen, aus der Krankenhauspraxis heraus untermauert werden.

Zentralisierung wird oftmals mit Kostensenkung assoziiert. Was spricht jenseits von Wirtschaftlichkeitsüberlegungen für eine Bündelung?

Schäfer-Jakobs: Jährlich stoßen rund 3400 Produktinnovationen in den Kliniksektor. Ein einzelnes Haus wäre überfordert, die neuen Medizinprodukte und Arzneimittel zu bewerten. Wir brauchen deswegen zentrale Strukturen und ein systematisches Bewertungsverfahren, ähnlich wie es andere Branchen auch handhaben. So können wir erreichen, dass das Wissen der Experten möglichst vielen Mitarbeitern zugänglich wird. Dies betrifft Kaufentscheidungen ebenso wie Aspekte, die Patientensicherheit gefährden und über diesen Evaluationsprozess schneller eliminiert werden können.

Wie gewichten Sie den Stellenwert von Preis und Qualität und Nutzerorientierung? Schäfer-Jakobs: An Platz eins steht die Qualität, gefolgt von der Nutzerorientierung. Erst danach kommt der Preis. Noch besser wäre es, den Terminus Preis durch den umfassenderen Begriff der Wirtschaftlichkeit zu ersetzen. Im Zuge dessen haben die Sachkosten naturgemäß eine hohe Priorität. Aber auch die Prozesskosten, welche maßgeblich durch die Standardisierung beeinflusst werden, sollten stärker in die Betrachtung mit einfließen. Insgesamt gilt: Die Qualität im Portfolio entscheidet über eine breite Akzeptanz beim Anwender und beim Patienten.

Frau Schäfer-Jakobs, vielen Dank für das Gespräch!

Das Interview führte Dr. Adelheid Weßling, freie Journalistin, Düsseldorf. 